\title{
Retos importantes para el establecimiento de una Ciudad Inteligente en Honduras
}

Fernando Zorto Aguilera ${ }^{1}$

Yeny Carías Alemán ${ }^{2}$

\section{RESUMEN}

Las ciudades alrededor del mundo tienen retos muy importantes para poder solventar diferentes aspectos para que los ciudadanos que viven dentro de ellas sean miembros de sociedades mucho más justas e inteligentes. El artículo discute los retos importantes que se deben afrontar para que la ciudad de Tegucigalpa pueda convertirse en una "smart city", basándose en el modelo de Lombardi (2012), el cual aplica el Proceso de Análisis de Red (ANP) para identificar si la ciudad cumple con los indicadores de ciudad inteligente.

\section{INTRODUCCIÓN}

El mundo está en la búsqueda de que las ciudades súper pobladas encuentren una manera de seguir siendo sustentables, dado que tienen un crecimiento muy rápido en los últimos años. La idea de estas ciudades es brindar las capacidades de ser una comunidad interconectada de manera interactiva con todos sus miembros para crear mecanismos de articulación y mejorar sus vidas plenamente.

Tegucigalpa es la capital de la República de Honduras, es una ciudad que está sumergida en múltiples problemas, siendo tres los más importantes: inseguridad, pobreza e infraestructura de saneamiento. Independientemente de sus problemas, la ciudad tiene sus oportunidades para cambiar a corto, mediano y largo plazo.

1 Profesor, Departamento de Ingeniería Mecánica, Facultad de Ingeniería, UNAH: fernando.zorto@unah.edu.hn

2 Profesora, Carrera de Ingeniería en Sistemas, Facultad de Ingeniería, UNAH: yenycarias@unah.edu.hn 


\section{Ciudad Inteligente}

La ciudades alrededor del mundo tienen un reto muy grande que abordar, dado que según los datos del Banco Mundial (2016), para el año 2030 el 60 \% de la población mundial vivirá en áreas urbanas. La preocupación de estos indicadores radica en que el crecimiento de estas ciudades va muy rápido, mostrando que más del $90 \%$ del crecimiento poblacional de los países en desarrollo ocurre en estas ciudades. Se estima que cada día que pasa se adicionan a las ciudades 180,000 personas a la población urbana de los países, de los cuales un tercio de esa población vivirá en dichas ciudades en situaciones de pobreza extrema y mucha desigualdad.

Este incremento de la población trasciende en múltiples problemáticas, algunas son la búsqueda de trabajo, educación, servicios públicos, infraestructura, comunicación, gestión, etc. Estos problemas radican, según Khatoun y Zeadally (2016), en la poca planificación de los arquitectos, quienes no consideraron la escalabilidad de acceso a los hogares, desarrollo sustentable, sistemas de transporte y crecimiento población. Asimismo, es necesario aclarar que no hay un sistema eficiente para la gestión administrativa de estas ciudades.

Khatoun y Zeadally (2016) manifiestan que las ciudades inteligentes deben solucionar estas complicaciones por medio de objetivos claves para crear este tipo de sociedades adelantadas: manejo optimizado de fuentes energéticas, descentralización de la producción de la energía, integración de los modelos de negocios y modelos económicos, seguridad, ambiente y transporte, administración de la energía residencial, inversión educativa, turismo y salud de los ciudadanos.

Por tal motivo, según Bakici y otros (2012), se define a una ciudad inteligente como una ciudad avanzada con alta tecnología intensiva, la cual conecta a las personas, la información y los elementos de la ciudad con nuevas tecnologías para crear una ciudad más sustentable, verde, competitiva e innovadora, ligado todo esto a un incremento en su calidad de vida.

Otros autores, como Gartner (2011), consideran que las ciudades inteligentes están basadas en el intercambio inteligente de información de los diferentes subsistemas que lo conforman. Es decir, existe un flujo de información que debe ser analizado por los usuarios para que se les brinden servicios a personas naturales y jurídicas. La masificación de este intercambio de información está basada en una estructura de gobierno electrónico diseñado para hacer una ciudad sustentable y autorregulable. 
Sin embargo, existen otros conceptos como los de Hall (2000) y Harrison y otros (2010), quienes manifiestan que la infraestructura civil como tecnológica debe ir de la mano para crear una ciudad en donde todos los procesos sean los más óptimos y consuman de manera sustentable sus recursos. Guan (2012) indica que al lograr estas condiciones se podrá generar un confort urbano pleno, en donde lo salud y la alegría de la comunidad sean las características más importantes para gestionar una ciudad cuyos ciudadanos y ambiente sean los pilares en los que se apoya la existencia de estas ciudades. Kourtit y otros (2012) plantea que para lograr estos pilares fundamentales planteados por Guan (2012), es necesario educar a las personas al más alto nivel posible para incrementar la innovación en la resolución de problemas en la comunidad donde ellos estarán viviendo.

Marsal-Llacuna et al (2014) acoplan lo antes descrito de una forma muy simple: "Las iniciativas de Smart Cities intentan mejorar los desarrollos urbanos por medio del uso de datos, información, tecnología de la información para brindar servicios más eficientes para los ciudadanos, monitorear y optimizar la infraestructura existente, incrementar la colaboración económica de los diferentes actores del mercado y alentar nuevos modelos de negocios para el sector público como privado ".

\section{Tegucigalpa: Situación Actual}

Entre algunos de los problemas identificados en ciudades como Tegucigalpa se pueden enumerar estos: personas viviendo de la recolección de basura, poco abastecimiento de agua, ya que las cuencas que alimentan a las cuencas ya están en su fase crítica, especialmente cuando hay pocas lluvias; deficiencias en el acceso a las calles y medios de transporte, el tráfico vehicular, el alumbrado público escaso que desencadena en inseguridad ciudadana, el manejo de desastres, entre muchos otros. En consecuencia, es necesario conocer los problemas, pero no solo para quejarse, sino para conocerlos y aportar soluciones alternativas, adentrarse en la ciudad, realizar una adecuada planificación urbana y hacer propuestas de mejora para tal o cual dificultad; sin olvidar de qué necesita la gente, qué es lo que la gente piensa y qué es lo que debemos hacer para cambiar el rumbo.

Hace 60 años, Tegucigalpa era una ciudad con poca población, pero hoy es una ciudad de más de un millón y medio de habitantes, con el aumento de la población, el incremento de los problemas y el desencadenamiento de una inconciencia en el manejo de los sistemas a todos niveles. Toda persona tiene derecho a circular libremente y a elegir su residencia en el territorio de cada Estado, pero es el gobierno el encargado de procurar las condiciones de seguridad necesarias. El concepto de 
espacio público que tiene que ver con la ciudad, también incluye el acceso a la educación, la salud, la seguridad, etc.

La palabra espacio se define como el conjunto de relaciones de simultaneidad o de coexistencia, es el resultado de las relaciones literarias, sociales, políticas, científicas, folklóricas, culturales, cosmogónicas, etc.; de allí que los ciudadanos tengan acceso a utilizar los espacios públicos a través de la recreación.

Un importante conjunto de relaciones de coexistencia como el espacio de convivencia que garantiza la reproducción de la vida, ha ido desarrollando las distintas mediaciones como los métodos, teorías, religiones, mitos, producción de bienes y servicios, mediaciones, etc., para lograr la construcción de este espacio de supervivencia. Recordemos que la supervivencia pasa por la convivencia de todos los humanos entre sí y su convivencia con la naturaleza.

\section{Tegucigalpa: Retos para ser una Smart City}

Para Kominos (2002) queda claro que existen cuatro dimensiones básicas para que cualquier ciudad se convierta realmente en una "smart city". La primera de ellas trata de la aplicación masiva de dispositivos electrónicos y digitales para crear realmente una línea de conocimiento base para la ciudad; la segunda es usar esta información tecnológica para transformar vida y trabajos; la tercera es desarrollar una infraestructura de ICT en toda la ciudad y la cuarta dimensión se encuentra orientada o dirigida a tomar la ICT y las personas de manera conjunta para aumentar la innovación, el aprendizaje y el conocimiento.

En Tegucigalpa, las dimensiones que se plantearon anteriormente se muestran un panorama poco alentador para que la capital pueda desarrollar de manera plena una iniciativa de ciudad inteligente. Es necesario medir a Tegucigalpa según los 60 indicadores de Lombardi (2012), lo cuales proponen una manera garantizada para cumplir y tener un punto de partida para mejorar dichos indicadores. El autor, los agrupa en las siguientes dimensiones: economía inteligente; ciudadanos inteligentes, gobierno inteligente, ambiente inteligente y viviendo inteligente. Queda claro que Tegucigalpa no cumple con estos indicadores. 


\section{Figura 1. Modelo para la conversión de Tegucigalpa a un Smart City}

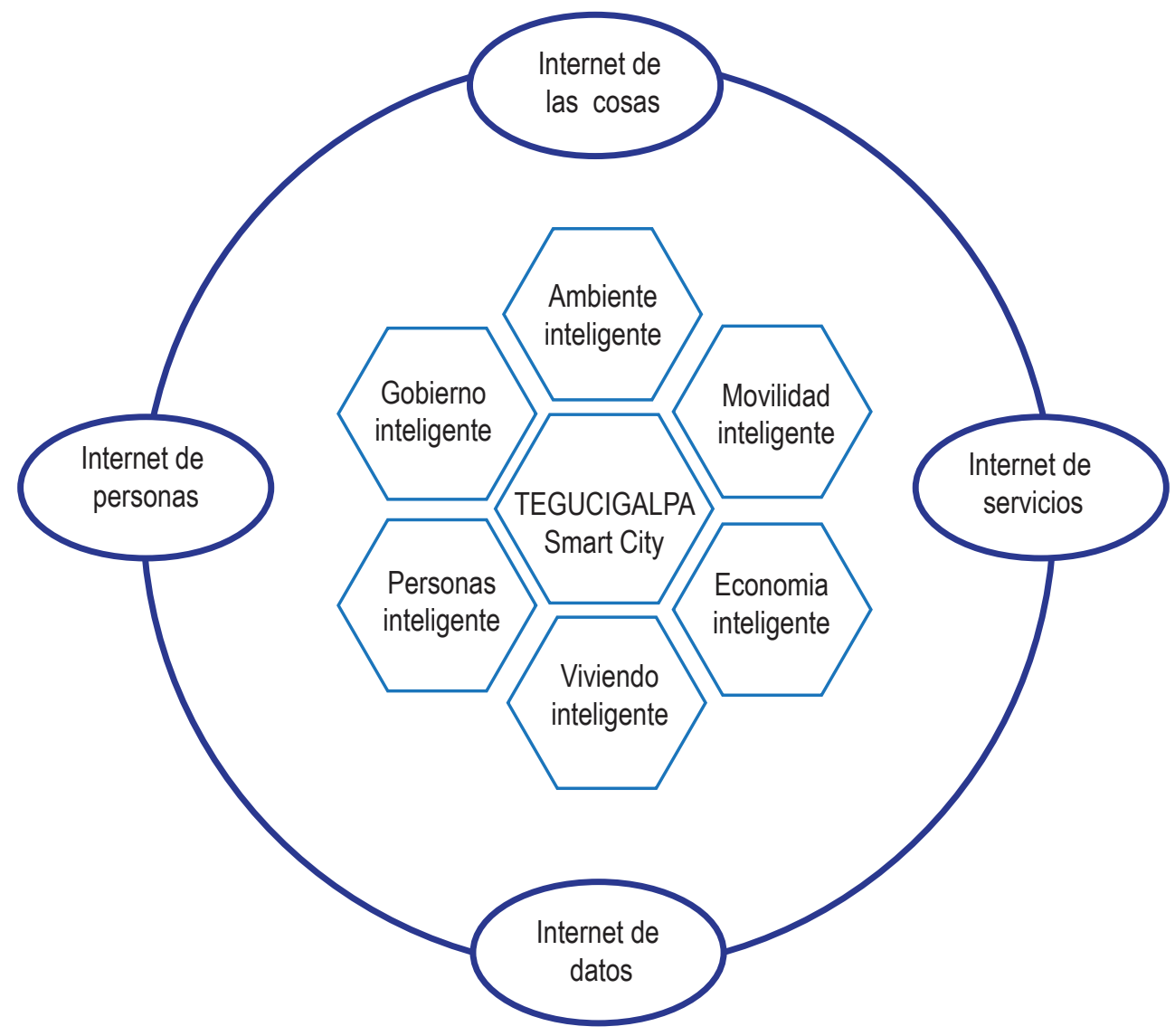

Fuente: Elaboración Propia

La figura 1 muestra el reto que tiene la municipalidad de Tegucigalpa para lograr avanzar en este camino de desarrollo de la ciudad como tal. Es necesario ver todas las aristas que se toman en cuenta y las diferentes conectividades que tiene el usuario de la capital al momento de usar el servicio de internet y como este conocimiento genera una mejor vida para todos los miembros de la comunidad.

\section{Hacktegus: una iniciativa de oportunidades}

El "hackaton" de innovación ciudadana de Tegucigalpa fue un evento para generar soluciones innovadoras para la ciudad usando datos abiertos, en donde la academia se vio representada a través del apoyo de la Universidad Nacional Autónoma de Honduras. El evento estuvo organizado de manera conjunta con el Ayuntamiento de 
Tegucigalpa y el Banco Interamericano de Desarrollo. Está enmarcado dentro de la Iniciativa de Ciudades Emergentes y Sostenibles del Banco Interamericano de Desarrollo, de la cual Tegucigalpa forma parte.

El propósito principal era desarrollar soluciones innovadoras para la ciudad utilizando datos abiertos, en donde los participantes debían proponer soluciones a la problemática de la ciudad de Tegucigalpa, en especial, se estaban buscando proyectos para las áreas de:

1. Comercio: ¿Cómo revitalizar el comercio en la ciudad?

2. Desastres naturales: ¿Cómo mitigar el efecto de los desastres naturales?

3. Saneamiento: ¿Cómo hacer nuestro río más saludable?

4. Seguridad urbana: ¿Cómo mejorar la seguridad en nuestra ciudad?

\section{CONCLUSIONES}

Haciendo una comparación entre ciudades de tercer y primer mundo, se pueden establecer grandes diferencias, entre ellas: el uso del transporte, el cual a pesar de parecer un solo ítem de la lista conlleva enraizado muchos otros aspectos: temas de seguridad ciudadana y contaminación ambiental. Recuperar los centros históricos y los espacios públicos va más allá de su utilización para el deleite del turismo externo e interno, constituyen el acceso a la historicidad de la ciudad y al salvamento de una cultura e identidad nacional propia; igualmente, la forma en cómo educamos al ciudadano para que forme parte del engrane de individuos que se desarrollan como motor de la economía y los procesos de intercomunicación con todos los sectores de la población para que se encuentren interconectados unos con otros.

\section{BIBLIOGRAFÍA}

Banco Mundial. (2016). Datos y cifras. Recuperado de: http://www.bancomundial.or$\mathrm{g} /$ temas/cities/datos.htm

BakıcI, T.; Almirall, E. and Wareham, J. (2012). A Smart City Initiative: The Case of Barcelona. Journal of the Knowledge Economy, 2, 11-14.

Guan, L. (2000). Smart Steps To A Battery City. Government News, 32, 2, 24-27. 
Hall, Robert. (2000). The Vision of a Smart City. Proc. of the 2nd International Life Extension Technology Workshop. Paris.

Harrison, C.; Eckman, B.; Hamilton, R.; Hartswick, P.; Kalagnanam, J.; Paraszczak, J. and Williams, P. (2010). Foundations for Smarter Cities. IBM Journal of Research and Development, 54, 4 1-16.凶

Khatoun, R. \& Zeadally, S. (2016). Smart cities: concepts, architectures, research opportunities. Communications of the ACM, 59(8), 46-57.

Komninos, N. (2002). Intelligent Cities: Innovation, Knowledge Systems and Digital Spaces. London: Spon Press.

Kourtit, K. and Nijkamp, P. (2012). Smart Cities in the Innovation Age. Innovation: The European Journal of Social Science Research, 25(2), 93-95.凶

Lombardi, P.; Giordano, S. Farouh, H. and Yousef, W. (2012). Modelling the Smart City Performance. Innovation: The European Journal of Social Science Research, 25(2), 137-149. 